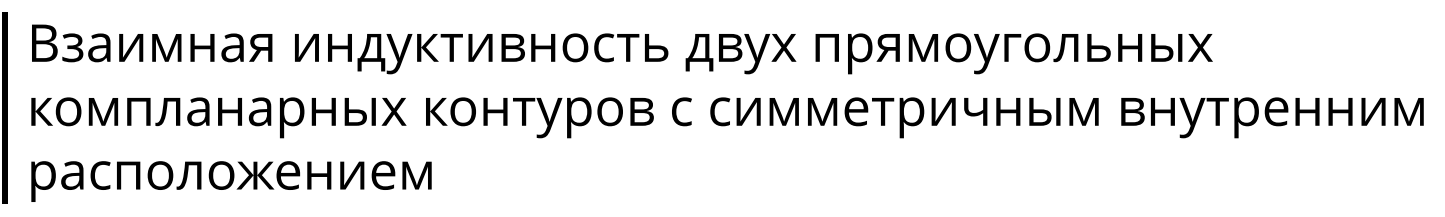

\begin{abstract}
Аннотация. Целесообразность вывода выражений для взаимной индуктивности прямоугольных и квадратных компланарных контуров обусловлена конфигурачией рамочных антенн, характерных для RFID-технологии. Представленные в данной статье выражения направлены на упрощение расчета коэффициентов индукции при проектировании рамочных антенн. Получены выражения для взаимной индуктивности прямоугольных компланарных контуров с симметричным внутренним расположением на основе метода участков. Показано, что полученные выражения при определенных условиях могут быть сведены к собственным индуктивностям квадратных и прямоугольных рамок, встречающихся в конфигурациях антенн. Предпринятый анализ позволяет оценивать области резонансных частот рамочных приемных антенн. Одновременно произведен сопоставительный анализ ряда выражений для собственной индуктивности прямоугольных и квадратных рамок и обращено внимание на различие в оценках. Даны рекомендации по уточнению внешней индуктивности квадратного контура.
\end{abstract}

Ключевые слова: коэффициенты само- и взаимоиндукции, компланарные контуры, рамочные антенны

Для цитирования: Цицикян Г. Н., Сенченко А. И. Взаимная индуктивность двух прямоугольных компланарных контуров с симметричным внутренним расположением // Изв. вузов России. Радиоэлектроника. 2018. № 3. С. $42-47$.

G. N. Tsitsikyan, A. I. Senchenko

Central Scientific Research Institute of Marine Electrical Engineering and Technology

"Krylov State Research Center"

6, Blagodatnaya Str., 196128, St. Petersburg, Russia

\title{
Mutual Inductance of Two Coplanar Rectangular Contours with Symmetrical Internal Arrangement
}

Abstract. The expediency of developing expressions for mutual inductance of rectangular and square coplanar contours is caused by configuration of frame antennas typical for RFID-technologies. Expressions presented in this article are aimed at simplifying calculation of induction coefficients when designing loop antennas. Expressions for mutual inductance of rectangular coplanar contours with symmetrical internal arrangement are obtained on the basis of the method of sections. It is shown that under certain conditions the expressions obtained can be reduced to intrinsic inductances of the square and rectangular frames encountered in antenna configurations. The analysis allows to estimate regions of resonance frequencies of frame receiving antennas. Simultaneously, a comparative analysis of a number of expressions for the intrinsic inductance of rectangular and square frames is performed, and attention is drawn to the difference in estimates. Recommendations for clarifying square contour external inductance are provided.

Keywords: coefficients of self and mutual induction, coplanar circuits, loop antenna.

For citation: Tsitsikyan G. N., Senchenko A. I. Mutual Inductance of Two Coplanar Rectangular Contours with Symmetrical Internal Arrangement. Izvestiya Vysshikh Uchebnykh Zavedenii Rossii. Radioelektronika [Journal of the Russian Universities. Radioelectronics]. 2018, no. 3, pp. 42-47. (In Russian)

Введение. Радиочастотная идентификация (RFID - Radio Frequency Identification) - это стремительно развивающаяся технология, позволяющая производить бесконтактную передачу информации об объекте, которая хранится в памяти прикрепляемой к нему радиочастотной метки. RFID открывает широкие возможности по созданию автоматических систем на производстве 
и во многих других областях современной жизни. Целесообразность вывода выражений для взаимной индуктивности прямоугольных и квадратных компланарных контуров обусловлена конфигурацией рамочных антенн, характерных для RFIDтехнологии [1]-[3].

Постановка задачи. Представленные в данной статье выражения направлены на упрощение расчета коэффициентов индукции при проектировании рамочных антенн. Их значения определяют область резонансных частот рамочных антенн различной конфигурации, в том числе для приемной рамочной антенны трансформаторного типа, показанной на рис. 1 , где $C_{1}, C_{2}$ переменные емкости. В настоящей статье выводятся выражения для коэффициентов само- и взаимной индукции рамочных антенн, применяемых в RFID-технологии.

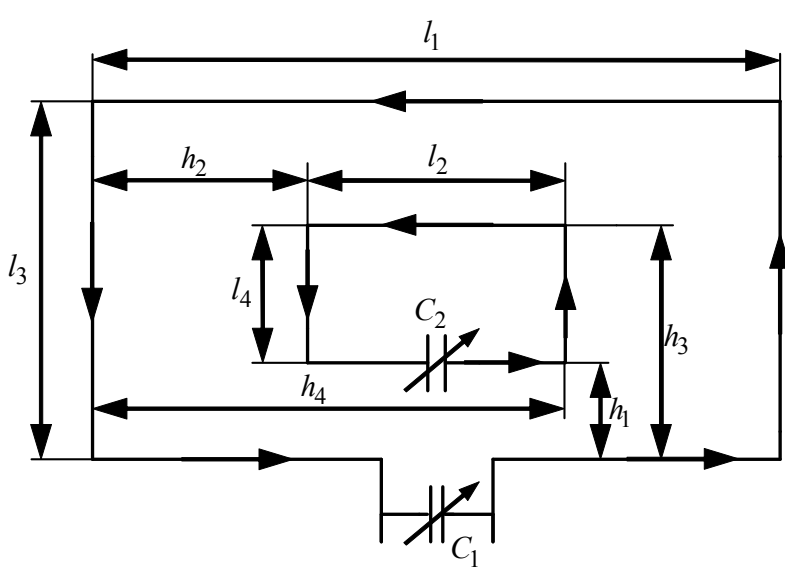

Puc. 1

Основная часть. На рис. 2 показано симметричное расположение отрезка $l_{2}$ относительно $l_{1}$. Для коэффициента взаимоиндукции $M_{1}$ при симметричном расположении отрезков $l_{1}$ и $l_{2}$, когда $l_{2}^{\prime}=$ $=\left(l_{1}-l_{2}\right) / 2$, имеем по методу участков согласно [4]: $M_{1}=\frac{\mu_{0}}{4 \pi}\left(\left(\frac{l_{1}-l_{2}}{2}\right) \ln \left\{-\frac{l_{1}-l_{2}}{2}+\left[\left(\frac{l_{1}-l_{2}}{2}\right)^{2}+h_{1}^{2}\right]^{1 / 2}\right\}+\right.$

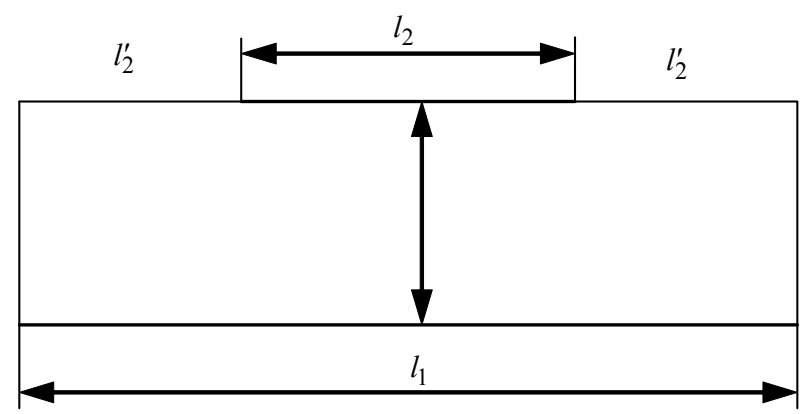

Puc. 2

$$
\begin{gathered}
+\left(\frac{l_{1}+l_{2}}{2}\right) \ln \left\{\frac{l_{1}+l_{2}}{2}+\left[\left(\frac{l_{1}+l_{2}}{2}\right)^{2}+h_{1}^{2}\right]^{1 / 2}\right\}- \\
-\left(\frac{l_{1}+l_{2}}{2}\right) \ln \left\{-\frac{l_{1}+l_{2}}{2}+\left[\left(\frac{l_{1}+l_{2}}{2}\right)^{2}+h_{1}^{2}\right]^{1 / 2}\right\}- \\
-\left(\frac{l_{1}-l_{2}}{2}\right) \ln \left\{\frac{l_{1}-l_{2}}{2}+\left[\left(\frac{l_{1}-l_{2}}{2}\right)^{2}+h_{1}^{2}\right]^{1 / 2}\right\}+ \\
\left.+2\left[\left(\frac{l_{1}-l_{2}}{2}\right)^{2}+h_{1}^{2}\right]^{1 / 2}-2\left[\left(\frac{l_{1}+l_{2}}{2}\right)^{2}+h_{1}^{2}\right]^{1 / 2}\right\}= \\
+\frac{\mu_{0}}{4 \pi}\left\{h_{2} \ln \left[-h_{2}+\left(h_{2}^{2}+h_{1}^{2}\right)^{1 / 2}\right]+\right. \\
+h_{4} \ln \left[h_{4}+\left(h_{4}^{2}+h_{1}^{2}\right)^{1 / 2}\right]-h_{4} \ln \left[-h_{4}+\left(h_{4}^{2}+h_{1}^{2}\right)^{1 / 2}\right]- \\
-h_{2} \ln \left[h_{2}+\left(h_{2}^{2}+h_{1}^{2}\right)^{1 / 2}\right]+2\left(h_{2}^{2}+h_{1}^{2}\right)^{1 / 2}- \\
\left.-2\left(h_{4}^{2}+h_{1}^{2}\right)^{1 / 2}\right\}=\frac{\mu_{0}}{2 \pi}\left\{h_{4} \ln \left[\frac{h_{4}+\left(h_{4}^{2}+h_{1}^{2}\right)^{1 / 2}}{h_{1}}\right]-\right. \\
-h_{2} \ln \left[\frac{h_{2}+\left(h_{2}^{2}+h_{1}^{2}\right)^{1 / 2}}{h_{1}}\right]+\left(h_{2}^{2}+h_{1}^{2}\right)^{1 / 2}- \\
\left.-\left(h_{4}^{2}+h_{1}^{2}\right)^{1 / 2}\right\},
\end{gathered}
$$

где $h_{2}=\frac{l_{1}-l_{2}}{2} ; h_{4}=\frac{l_{1}+l_{2}}{2} ; \mu_{0}-$ магнитная постоянная.

На рис. 3 показано симметричное расположение отрезка $l_{4}$ относительно $l_{3}$. Для записи коэффициента взаимоиндукции отрезков $l_{3}$ и $l_{4}$ достаточно $l_{1}$ заменить на $l_{3}, l_{2}$ - на $l_{4}$ и $h_{1}$ заменить на $h_{2}$ :

$$
\begin{gathered}
M_{2}=\frac{\mu_{0}}{4 \pi}\left(\left(\frac{l_{3}-l_{4}}{2}\right) \times\right. \\
\times \ln \left\{-\frac{l_{3}-l_{4}}{2}+\left[\left(\frac{l_{3}-l_{4}}{2}\right)^{2}+h_{2}^{2}\right]^{1 / 2}\right\}+
\end{gathered}
$$

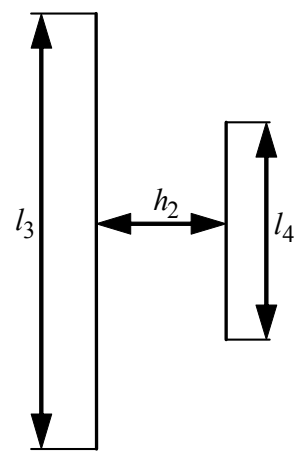

Puc. 3 


$$
\begin{gathered}
+\left(\frac{l_{3}+l_{4}}{2}\right) \ln \left\{\frac{l_{3}+l_{4}}{2}+\left[\left(\frac{l_{3}+l_{4}}{2}\right)^{2}+h_{2}^{2}\right]^{1 / 2}\right\}- \\
-\left(\frac{l_{3}+l_{4}}{2}\right) \ln \left\{-\frac{l_{3}+l_{4}}{2}+\left[\left(\frac{l_{3}+l_{4}}{2}\right)^{2}+h_{2}^{2}\right]^{1 / 2}\right\}- \\
-\left(\frac{l_{3}-l_{4}}{2}\right) \ln \left\{\frac{l_{3}-l_{4}}{2}+\left[\left(\frac{l_{3}-l_{4}}{2}\right)^{2}+h_{2}^{2}\right]^{1 / 2}\right\}+ \\
\left.+2\left[\left(\frac{l_{3}-l_{4}}{2}\right)^{2}+h_{2}^{2}\right]^{1 / 2}-2\left[\left(\frac{l_{3}+l_{4}}{2}\right)^{2}+h_{2}^{2}\right]^{1 / 2}\right\}= \\
=\frac{\mu_{0}}{4 \pi}\left\{h_{1} \ln \left[-h_{1}+\left(h_{1}^{2}+h_{2}^{2}\right)^{1 / 2}\right]+\right. \\
+h_{3} \ln \left[h_{3}+\left(h_{3}^{2}+h_{2}^{2}\right)^{1 / 2}\right]- \\
-h_{3} \ln \left[-h_{3}+\left(h_{3}^{2}+h_{2}^{2}\right)^{1 / 2}\right]- \\
-h_{1} \ln \left[h_{1}+\left(h_{1}^{2}+h_{2}^{2}\right)^{1 / 2}\right]+ \\
\left.+2\left(h_{1}^{2}+h_{2}^{2}\right)^{1 / 2}-2\left(h_{3}^{2}+h_{2}^{2}\right)^{1 / 2}\right\}= \\
\left.+\left(h_{1}^{2}+h_{2}^{2}\right)^{1 / 2}-\left(h_{3}^{2}+h_{2}^{2}\right)^{1 / 2}\right\} \\
=\frac{\mu_{0}}{2 \pi}\left\{h_{3} \ln \left[\frac{h_{3}+\left(h_{3}^{2}+h_{2}^{2}\right)^{1 / 2}}{h_{2}}\right]-\right. \\
{\left[\frac{h_{1}+\left(h_{1}^{2}+h_{2}^{2}\right)^{1 / 2}}{h_{2}}\right]+}
\end{gathered}
$$

где $h_{1}=\frac{l_{3}-l_{4}}{2} ; h_{3}=\frac{l_{3}+l_{4}}{2}$.

Для записи коэффициентов $M_{3}$ и $M_{4}$ следует в (1) заменить $h_{1}$ на $h_{3}$, в (2) $h_{2}$ на $h_{4}$ и изменить знаки на противоположные. Тогда получим:

$$
\begin{gathered}
M_{3}=-\frac{\mu_{0}}{2 \pi}\left\{h_{4} \ln \left[\frac{h_{4}+\left(h_{4}^{2}+h_{3}^{2}\right)^{1 / 2}}{h_{2}}\right]-\right. \\
-h_{2} \ln \left[\frac{h_{2}+\left(h_{2}^{2}+h_{3}^{2}\right)^{1 / 2}}{h_{3}}\right]+\left(h_{2}^{2}+h_{3}^{2}\right)^{1 / 2}- \\
\left.-\left(h_{4}^{2}+h_{3}^{2}\right)^{1 / 2}\right\} ; \\
M_{4}=-\frac{\mu_{0}}{2 \pi}\left\{h_{3} \ln \left[\frac{h_{3}+\left(h_{3}^{2}+h_{4}^{2}\right)^{1 / 2}}{h_{4}}\right]-\right.
\end{gathered}
$$

$$
\begin{gathered}
-h_{1} \ln \left[\frac{h_{1}+\left(h_{1}^{2}+h_{4}^{2}\right)^{1 / 2}}{h_{4}}\right]+\left(h_{1}^{2}+h_{4}^{2}\right)^{1 / 2}- \\
\left.-\left(h_{3}^{2}+h_{4}^{2}\right)^{1 / 2}\right\} .
\end{gathered}
$$

Искомый коэффициент взаимоиндукции будет равен двухкратной сумме коэффициентов от $M_{1}$ до $M_{4}$ :

$$
M=2 \sum_{n=1}^{4} M_{n} .
$$

Интересен результат для случая, когда $l_{1}=l_{3}$ и $l_{2}=l_{4}, h_{1}=h_{2}$ и $h_{3}=h_{4}$, т. е. для рамок квадратной формы (рис. 4).

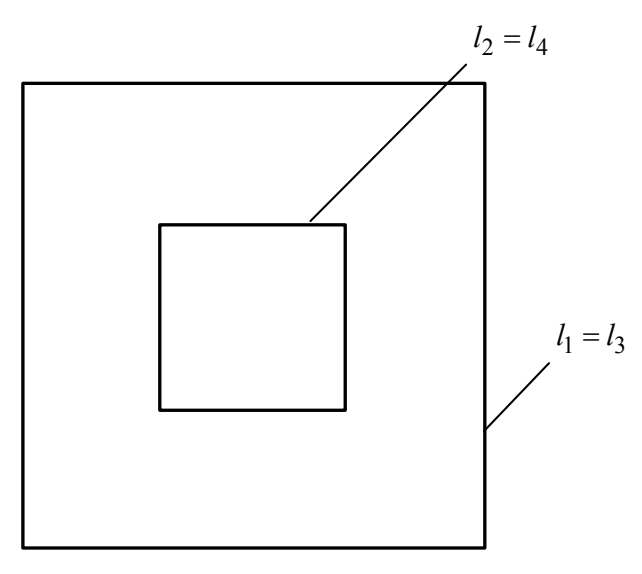

Puc. 4

Тогда $M_{1}=M_{2}, M_{3}=M_{4}$ и

$$
\begin{gathered}
M_{\text {кв }}=4\left(M_{1}+M_{3}\right)= \\
=\frac{2 \mu_{0}}{\pi}\left\{h_{4} \ln \left[\frac{h_{4}+\left(h_{4}^{2}+h_{1}^{2}\right)^{1 / 2}}{h_{1}}\right]-\right. \\
-h_{2} \ln \left[\frac{h_{2}+\left(h_{2}^{2}+h_{1}^{2}\right)^{1 / 2}}{h_{1}}\right]+ \\
+\left(h_{2}^{2}+h_{1}^{2}\right)^{1 / 2}-\left(h_{4}^{2}+h_{1}^{2}\right)^{1 / 2}- \\
-h_{4} \ln \left[\frac{h_{4}+\left(h_{4}^{2}+h_{3}^{2}\right)^{1 / 2}}{h_{3}}\right]+ \\
+h_{2} \ln \left[\frac{h_{2}+\left(h_{2}^{2}+h_{3}^{2}\right)^{1 / 2}}{h_{3}}\right]- \\
\left.-\left(h_{2}^{2}+h_{3}^{2}\right)^{1 / 2}+\left(h_{4}^{2}+h_{3}^{2}\right)^{1 / 2}\right\}=
\end{gathered}
$$




$$
\begin{gathered}
=\frac{2 \mu_{0}}{\pi}\left\{h_{3} \ln \left[\frac{h_{3}+\left(h_{3}^{2}+h_{1}^{2}\right)^{1 / 2}}{h_{1}}\right]-\right. \\
-h_{1} \ln (1+\sqrt{2})+\sqrt{2} h_{1}- \\
-\left(h_{3}^{2}+h_{1}^{2}\right)^{1 / 2}-h_{3} \ln (1+\sqrt{2})+ \\
+h_{1} \ln \left[\frac{h_{1}+\left(h_{1}^{2}+h_{3}^{2}\right)^{1 / 2}}{h_{3}}\right]- \\
\left.-\left(h_{1}^{2}+h_{3}^{2}\right)^{1 / 2}+\sqrt{2} h_{3}\right\}= \\
=\frac{2 \mu_{0} h_{3}}{\pi}\left(\ln \left\{\frac{h_{3}}{h_{1}}\left[1+\left(1+\frac{h_{1}}{h_{3}}\right)^{2}\right]^{1 / 2}\right\}-\right. \\
-\left(1+\frac{h_{1}}{h_{3}}\right) \ln (1+\sqrt{2})+\sqrt{2}\left(1+\frac{h_{1}}{h_{3}}\right)- \\
-2\left[\left(1+\frac{h_{1}}{h_{3}}\right)^{2}\right]^{1 / 2}+ \\
\left.+\frac{h_{1}}{h_{3}} \ln \left\{\frac{h_{1}}{h_{3}}\left[1+\left(1+\frac{h_{3}}{h_{1}}\right)^{2}\right]^{1 / 2}\right\}\right) .
\end{gathered}
$$

Полагая, например, в (3) $\frac{h_{1}}{h_{3}}=0.5$, получим:

$$
\begin{gathered}
M_{\text {Кв }}=\frac{2 \mu_{0} h_{3}}{\pi}(1.4436-1.3221+2.1213- \\
-2.2361+0.2406)=\frac{2 \mu_{0} h_{3}}{\pi} 0.4946 .
\end{gathered}
$$

В приближении $h_{1} / h_{3}=1$ для $M_{\text {кв }}$ будем иметь в соответствии с (3):

$$
\begin{gathered}
\left.M_{\text {кв }}\right|_{h_{1}=h_{3}} \cong \frac{2 \mu_{0}}{\pi} h_{3}\left[\ln \left(\frac{2 h_{3}}{h_{1}}\right)-\right. \\
-\ln (\sqrt{2}+1)+\sqrt{2}-2]= \\
=\frac{2 \mu_{0}}{\pi} h_{3}\left(\ln \frac{2 h_{3}}{h_{1}}-0.8814+1.4142-2\right)= \\
=\frac{2 \mu_{0}}{\pi} h_{3}\left(\ln \frac{2 h_{3}}{h_{1}}-1.4672\right) \cong \\
\cong \frac{2 \mu_{0}}{\pi} h_{3}\left(\ln \frac{h_{3}}{h_{1}}-0.774\right) .
\end{gathered}
$$

С целью дальнейших сопоставлений обратим внимание на выражение для внешней индуктивности квадратного контура, образованного внутренним контуром и контуром штриховой линии, представленными на рис. 5 (число витков

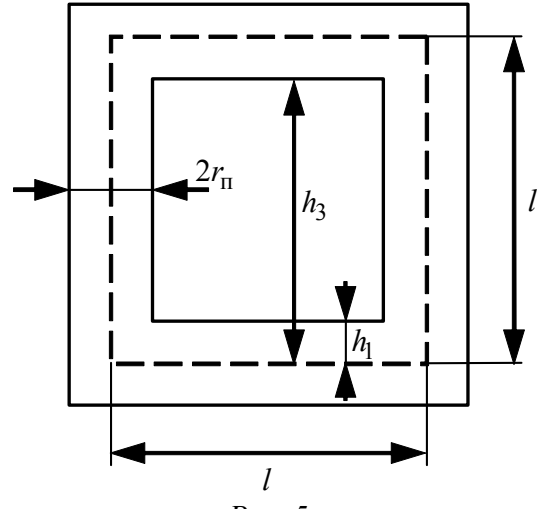

Puc. 5

$w=1.0, \quad r_{\Pi}$ - радиус провода). При $r_{\Pi}=l$ взаимная индуктивность между этими контурами и внешняя индуктивность рамки будут весьма близки друг к другу и (4), с учетом смены обозначений $h_{3} \cong l$ и $h_{1}=r_{\Pi}$, можно записать в виде

$$
M_{\text {Кв }}=L_{\mathrm{BH}}=\frac{2 \mu_{0} l}{\pi}\left(\ln \frac{l}{r_{\Pi}}-0.774\right),
$$

которая близка к рекомендуемой в [5] и [6] для определения внешней индуктивности квадратной рамки (рис. 5) со стороной $l$ и радиусом провода $r_{\text {п }}$.

Если учитывать еще и внутреннюю индуктивность провода радиуса $r_{\text {П }}$, то для (5) имеем:

$$
L=\frac{2 \mu_{0} l}{\pi}\left(\ln \frac{l}{r_{\Pi}}-0.524\right)
$$

Если в (6) подставлять линейные размеры в сантиметрах, то в полном соответствии с [7] получим:

$$
\begin{aligned}
L= & 0.008 l\left(\ln \frac{l}{r_{\Pi}}-0.774+0.25\right)= \\
= & 0.008 l\left(\ln \frac{l}{r_{\Pi}}-0.524\right) .
\end{aligned}
$$

С целью дальнейших сопоставлений воспользуемся выражением для внешней самоиндукции прямоугольной рамки одинаковой ширины

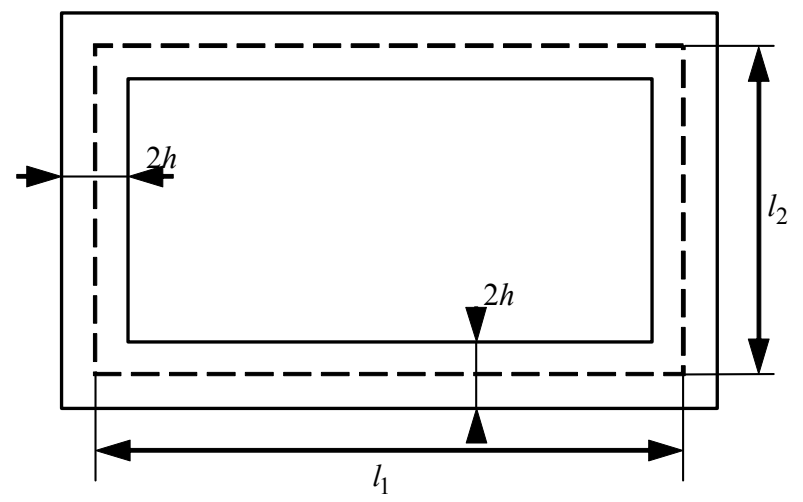

Puc. 6 
$2 h=2 r_{\text {п }}$ (рис. 6), как в [6] и в [8], а также приведенным в [2], только с той разницей, что линейные размеры даны в сантиметрах, что, конечно, несущественно.

Выражение для $L_{\text {вн }}$ во всех случаях сводится к виду

$$
\begin{aligned}
L_{\mathrm{BH}} \cong & \frac{\mu_{0}}{\pi}\left\{l_{1} \ln \frac{2 l_{1}}{r_{\Pi}}+l_{2} \ln \frac{2 l_{2}}{r_{\Pi}}-\right. \\
& -l_{1} \ln \frac{l_{1}+\left(l_{1}^{2}+l_{2}^{2}\right)^{1 / 2}}{l_{2}}- \\
& -l_{2} \ln \frac{l_{2}+\left(l_{2}^{2}+l_{1}^{2}\right)^{1 / 2}}{l_{1}}+ \\
+2 r_{\Pi} & \left.-2\left(l_{1}+l_{2}\right)+2\left[\left(l_{1}^{2}+l_{2}^{2}\right)^{1 / 2}\right]\right\}= \\
= & \frac{\mu_{0}}{\pi}\left\{l_{1} \ln \frac{2 l_{1} l_{2}}{r_{\Pi}\left[l_{1}+\left(l_{2}^{2}+l_{1}^{2}\right)^{1 / 2}\right]}+\right. \\
+ & l_{2} \ln \frac{2 l_{1} l_{2}}{r_{\Pi}\left[l_{2}+\left(l_{1}^{2}+l_{2}^{2}\right)^{1 / 2}\right]}+ \\
+ & \left.2\left(l_{1}^{2}+l_{2}^{2}\right)^{1 / 2}-2\left(l_{1}+l_{2}-r_{\Pi}\right)\right\} .
\end{aligned}
$$

При $l_{1}=l_{2}=l$ из последнего выражения получаем:

$$
\begin{aligned}
\left.L_{\mathrm{BH}}\right|_{l_{1}=l_{2}=l} & =\frac{2 \mu_{0} l}{\pi}\left\{\ln \frac{2 l}{r_{\Pi}(1+\sqrt{2})}+\sqrt{2}-2+\frac{r_{\Pi}}{l}\right\}= \\
& =\frac{2 \mu_{0} l}{\pi}\left(\ln \frac{l}{r_{\Pi}}-0.774+\frac{r_{\Pi}}{l}\right),
\end{aligned}
$$

что практически не отличается от (4).

Заключение. Из изложенного вытекает, что результат вывода для взаимной индукции компланарных прямоугольных контуров, один из которых симметрично располагается внутри другого, не противоречит выражению для внешней индуктивности $L_{\text {вн }}$ прямоугольной рамки, когда расположение внутреннего контура эквидистантно и расстояние между контурами весьма мало $\left(2 r_{\Pi}=l\right)$. При проектировании устройств могут возникнуть затруднения, вызванные отсутствием в ряде случаев выражений для расчета индуктивных параметров прямоугольных контуров, в частности, расположенных в одной плоскости. В связи с этим ключевую роль приобретают расчетные выражения для определения индуктивностей. Подчеркнем, что предпринятый анализ позволяет так же оценивать области резонансных частот рамочных приемных антенн. Собственные частоты, при которых в них наступает резонанс, в случае отсутствия взаимной индукции равны: $\omega_{1}=1 / \sqrt{L_{1} C_{1}}$ и $\omega_{2}=1 / \sqrt{L_{2} C_{2}}$.

Выражение для резонансных частот в индуктивно связанных контурах дано в [9].

\section{СПИСОК ЛИТЕРАТУРЫ}

1. Lee Y. RFID Coil Design. Microchip AN678 / Microchip Technology Inc. Chandler, USA, 1998. P. DS00678B (1-18).

2. Lee Y. Antenna Circuit Design for RFID Applications. Microchip AN710 / Microchip Technology Inc. Chandler, USA, 2003. P. DS00710C (1-50).

3. RFID Technology Principles, Advantages, Limitations and its Applications / M. Kaur, M. Sandhu, N. Mohan, P.S. Sandhu // Intern. J. of computer and Electrical Engineering. 2011. Vol. 3, № 1. Р. 151-157.

4. Цицикян Г. Н. Электродинамические силы в токоведущих частях электротехнических комплексов / ФГУП "Крыловский государственный научный центр". СПб., 2016. 94 с.

Статья поступила в редакцию 14 ноября 2017 г.
5. Paul C. R. Inductance: Loop and Partial. New York: Wiley, 2011. 379 p.

6. Tesche F. H., Ianoz M., Karlsson T. EMC Analysis Methods and Computational Models. New York: Wiley, $1997,623 \mathrm{p}$.

7. Grover F. W. Inductance Calculations. Working Formulas and Tables / D. Van Nostrand Co. Inc. New York, 1947. 98 p.

8. Fujimoto K., Morishita H. Modern Small Antennas. Cambridge University Press, 2013. 488 p.

9. Нейман Л. Р., Демирчян К. С. Теоретические основы электротехники: учеб. для вузов: в 2 т. Л.: Энергоиздат, 1981. Т. 1.536 р.

Цицикян Георгий Николаевич - доктор технических наук (1990), профессор (2000), зам. начальника отдела, ученый секретарь НТС (филиал "ЦНИИ СЭТ" ФГУП "Крыловский государственный научный центр"). Автор более 150 научных работ. Сфера научных интересов - электродинамические силы; электромагнитная совместимость; качество электроэнергии в судовой электротехнике.

E-mail: george.20021940@mail.ru

Сенченко Аркадий Игоревич - специалист по направлению "Системы автоматизации и электроэнергетики" (2012, Санкт-Петербургский государственный морской технический университет), инженерконструктор 1-й категории (филиал "ЦНИИ СЭТ" ФГУП "Крыловский государственный научный центр"). Автор 10 научных работ. Сфера научных интересов - качество электроэнергии, электромагнитная совместимость. E-mail: senchenko.1990@inbox.ru 


\section{REFERENCES}

1. Lee Y. RFID Coil Design. Microchip AN678. Microchip Technology Inc. Chandler, USA, 1998, pp. DS00678B (1-18).

2. Lee Y. Antenna Circuit Design for RFID Applications. Microchip AN710. Microchip Technology Inc. Chandler, USA, 2003, pp. DS00710C (1-50).

3. Kaur M., Sandhu M., Mohan N., Sandhu P. S. RFID Technology principles, advantages, limitations and its Applications. International Journal of computer and Electrical Engineering. 2011, vol. 3, no. 1, pp. 151-157.

4. Tsitsikyan G. N. Elektrodinamicheskie sily $v$ tokovedushchikh chastyakh elektrotekhnicheskikh kompleksov [Electrodynamic Forces in Current-Carrying Parts of Electrotechnical Complexes]. SPb, 2016, 94 p. (In Russian)
5. Paul C. R. Inductance: Loop and Partial. New York, Wiley, 2011, 379 p.

6. Tesche F. H., Ianoz M., Karlsson T. EMC Analysis Methods and Computational Models. New York, Wiley, 1997, $623 \mathrm{p}$.

7. Grover F. W. Inductance Calculations. Working Formulas and Tables. D. Van Nostrand Co. Inc. New York, 1947, 98 p.

8. Fujimoto K., Morishita H. Modern Small Antennas. Cambridge University Press, 2013, 488 p.

9. Neiman L. R., Demirchyan K. S. Teoreticheskie osnovy elektrotekhniki [Theoretical Fundamentals of Electrical Engineering]. Leningrad, Energoizdat, 1981, vol. 1, 536 p. (In Russian)

Received November, 14, 2017

George N. Tsitsikian - D.Sc. in Engineering (1990), Professor (2000), Deputy Head of Department, scientific secretary of STC (branch of Central Scientific Research Institute of Marine Electrical Engineering and Technology "Krylov state research center"). The author of more than 150 scientific publications. Area of expertise: electrodynamic forces; electromagnetic compatibility; power quality in ship electrical engineering.

E-mail: george.20021940@mail.ru

Arkady I. Senchenko - specialist in automation and power systems (2012, St. Petersburg State Maritime Technical University), 1st category Design Engineer (branch of Central Scientific Research Institute of Marine Electrical Engineering and Technology "Krylov State Research Center"). The author of 10 scientific publications. Area of expertise: power quality, electro-magnetic compatibility.

E-mail: senchenko.1990@inbox.ru 